\title{
Critical Exponents of Inhomogeneous Ferromagnets
}

\author{
A. Berger, G. Campillo ${ }^{\dagger}$, P. Vivas ${ }^{\dagger}$, J. E. Pearson, and S. D. Bader \\ Materials Science Division, Argonne National Laboratory, Argonne, IL 60439, USA \\ E. Baca and P. Prieto \\ Departamento de Física, Universidad del Valle, Cali, A. A. 25360, Colombia
}

\begin{abstract}
A method for the analysis of temperature and field dependent magnetization data near the Curie temperature $T_{C}$ is devised, which allows the reliable determination of critical exponents even in strongly inhomogeneous ferromagnets. It is shown, both experimentally and theoretically, that the field-induced broadening of the ferromagnetic phase transition follows a power law with a characteristic critical exponent $\beta \cdot \delta$ where $\beta$ and $\delta$ are the conventional temperature and field dependent critical exponents of the magnetization. Experimental results on inhomogeneous $\mathrm{La}_{2 / 3} \mathrm{Ca}_{1 / 3} \mathrm{MnO}_{3}$ films yield critical exponents $\beta=0.3682 \pm 0.0033$ and $\delta=4.76 \pm 0.32$, even though the intrinsic $\mathrm{T}_{\mathrm{C}}$ distribution width is larger than the field-induced effect.
\end{abstract}

\section{Author for Correspondence:}

Dr. Andreas Berger

IBM Almaden Research Center

650 Harry Road, K63/E3

San Jose, CA 95120

Tel. (408) 9272514

FAX (408) 9273008

e-mail: aberger@us.ibm.com 
Understanding critical phenomena has long been a key issue in the study of magnetism [1]. In particular, the determination of critical exponents has been an important aspect of the theoretical description and experimental characterization of magnetic materials [2]. For the experimental study of critical phenomena, one is typically inclined to use almost perfect samples, because only that allows comparisons with the most advanced theories, which are based upon uniform material properties [3]. However, it is not always possible to achieve very uniform samples and in such cases one has to resort to approximate descriptions that can cope with sample inhomogeneities. The aspect of sample inhomogeneity is of particular significance in the field of manganite materials, because ferromagnetic samples are randomly doped mixed valence systems. Such ferromagnets are, therefore, intrinsically inhomogeneous on a short length scale and actual samples can exhibit inhomogeneities on length scales up to the sample size. Thus, it is desirable to devise a method that allows an accurate and straightforward determination of critical exponents in inhomogeneous ferromagnets. In this paper, we describe such a method and apply it to inhomogeneous $\mathrm{La}_{2 / 3} \mathrm{Ca}_{1 / 3} \mathrm{MnO}_{3}$ (LCMO) film samples.

$\mathrm{La}_{2 / 3} \mathrm{Ca}_{1 / 3} \mathrm{MnO}_{3}$ films were grown by $\mathrm{DC}$-sputtering from a 35 -mm diameter ceramic target. Stoichiometrically sintered $\mathrm{La}_{2 / 3} \mathrm{Ca}_{1 / 3} \mathrm{MnO}_{3}$ targets were prepared by solid-state synthesis from starting materials $\mathrm{La}_{2} \mathrm{O}_{3}, \mathrm{CaCO}_{3}$, and $\mathrm{MnO}_{2}$ of nominal composition. The mixture was first fired in air at $950^{\circ} \mathrm{C}$ for 12 hours to achieve decarbonation. Subsequently, the product was grinded, pressed into the form of a disk, and sintered at $1200^{\circ} \mathrm{C}$ for 24 hours in air. The pellets were then re-grinded and fired at $1200^{\circ} \mathrm{C}$ for another 24 hours, followed by a slow cool-down at a rate of $5^{\circ} \mathrm{C} / \mathrm{min}$. For the film deposition process, the disks were mounted onto one of the electrodes of the sputtering system. Pure oxygen at a pressure of $3.5 \mathrm{mbar}$ was used as sputtering gas, and a potential difference of $300 \mathrm{~V}$ with a current of $100 \mathrm{~mA}$ was applied between the electrodes. During deposition the substrate temperature was kept at $850^{\circ} \mathrm{C}$. For the present study, films of 200-nm thickness each were grown onto $\mathrm{SrTiO}_{3}(001)$ single crystal substrates. The deposition rate was kept constant at approximately $1.5 \mathrm{~nm} / \mathrm{min}$. for all films. No post-deposition annealing of the films was necessary. X-ray diffraction (XRD) measurements $(\theta-2 \theta)$ were performed using a Rigaku diffractometer $\left(\mathrm{Cu} \mathrm{K} \mathrm{K}{ }^{-}\right.$ 
line: $\lambda=1.5406 \AA$ ) and showed our films to have (002) orientation with bulk-like lattice parameters [4]. All magnetic measurements were made using a Quantum Design extraction magnetometer, equipped with a 7-T superconducting solenoid.

In a previous study, we investigated the sample homogeneity of sputter deposited LCMO films with a particular emphasis on the $\mathrm{T}_{\mathrm{C}}$ distribution [4]. We found it possible to describe the average temperature-dependent magnetization $\mathrm{M}(\mathrm{T})$ by a superposition of individual power-law functions according to

$$
M(T)=m_{0} \int_{T_{C}}\left(\frac{T_{C}-T}{T_{C}}\right)^{\beta} \theta\left(T_{C}-T\right) \cdot \rho\left(T_{C}\right) d T_{C} .
$$

Here, $\mathrm{m}_{0}$ is a factor proportional to the saturation magnetization and $\beta$ is the temperature critical exponent of the magnetization. $\theta(\mathrm{x})$ is the Heavyside function, which insures that the magnetization vanishes for $\mathrm{T}>\mathrm{T}_{\mathrm{C}}$ and $\rho\left(T_{C}\right)$ is a sample-specific $\mathrm{T}_{\mathrm{C}}$ distribution function. For our LCMO films, the distribution function is taken to be a Gaussian

$$
\rho\left(T_{C}, \bar{T}_{C}, \Delta T_{C}\right)=\frac{1}{\sqrt{2 \pi} \cdot \Delta T_{C}} \exp \left(-\frac{1}{2} \frac{\left(T_{C}-\bar{T}_{C}\right)^{2}}{\Delta T_{C}^{2}}\right)
$$

characterized by $\bar{T}_{C}$, the average Curie temperature and $\Delta T_{C}$, the Curie temperature distribution width.

Figure 1 shows two sets of experimental data, measured on the same film for different values of the applied field $\mathrm{H}$, in comparison to the fit-function according to Eq. (1) under the assumption of the Gaussian $T_{C}$ distribution Eq. (2). As one can see from the comparison in Fig. 1, the agreement is excellent. One also observes, that the transition width of the ferromagnetic phase transition increases with $\mathrm{H}$. This is not surprising, because even in an ideal ferromagnet, one expects the phase transition to broaden in a field. The fit parameter $\Delta T_{C}$, displayed in the inset of Fig. 1, is therefore a superposition 
of the intrinsic Curie temperature distribution $\Delta T_{C}{ }^{0}$ and a field-induced broadening effect. We find experimentally that the latter follows a power law:

$$
\Delta T_{C}(H)=\Delta T_{C}^{0}+c \cdot H^{\frac{1}{n}},
$$

with a characteristic exponent $\eta$ and a sample dependent constant $\mathrm{c}$. A least-squares fit of the $\Delta T_{C}(\mathrm{H})$-data to Eq. (3) is shown as a solid line in the inset of Fig. 1. In addition, we observe that the values determined for $\eta$ are in the range between $1.65-1.80$ for all our samples. From these experimental observations arise two questions: Is it theoretically expected that $\Delta T_{C}(\mathrm{H})$ follows a power-law and, if so, what is the relevance of $\eta$ ? The fact that $\eta$ is associated with applying a field near $T_{C}$, suggests that it is related to $\delta$, the critical exponent that describes the field dependence of $M\left(T_{C}\right)$ :

$$
M\left(H, T=T_{C}\right)=\tilde{m} \cdot H^{1 / \delta}
$$

with $\tilde{m}$ being a factor proportional to the saturation magnetization [2].

To investigate the relevance of $\eta$ and its relation to the various critical exponents, we have performed a theoretical evaluation of the field broadening of $\mathrm{M}(\mathrm{T})$ for a number of model systems. From the equation of state, $\mathrm{M}=\mathrm{M}(\mathrm{T}, \mathrm{H})$, which is documented in the literature for the mean-field model, and the two (2D) and three dimensional (3D) Ising models [5], we calculated a set of $\mathrm{M}(\mathrm{T})$ curves for a number of fixed magnetic field values $H_{f}$. Subsequently, we analyzed each of these $M\left(T, H=H_{f}\right)$ curves in the same way, in which we had analyzed our experimental data, i.e. fit them to Eq. (1) under the assumption of a Gaussian $\mathrm{T}_{\mathrm{C}}$ distribution. This procedure enables us to determine $\Delta T_{C}(\mathrm{H})$, i.e. the experimentally determined quantity, for the different theoretical models. The results of this analysis are shown in Fig. 2. As one can see from the data, all models exhibit a power law behavior for $\Delta T_{C}$ vs. $\mathrm{H}$, following Eq. (3) in the same way as our experimental results. Thus, the phenomenologically observed power law, Eq. (3), has a firm foundation, because its validity was confirmed for several theoretical models. The 
significance of $\eta$ becomes evident, if one compares the theoretically deduced values with other critical exponents, shown in Table 1. From this comparison it is evident that within the numerical accuracy achieved in this study

$$
\eta=\beta \cdot \delta
$$

for all three models considered. Thus, we see compelling evidence that $\eta$ is truly equal to $\beta \cdot \delta$, which in turn means that our $\Delta T_{C}(\mathrm{H})$ measurements allow the experimental determination of $\beta \cdot \delta$. Figure 3a shows experimental data for an LCMO film in comparison with the power-law fit according to Eq. (3). To visualize the power-law behavior of the experimental data, we subtracted the intrinsic width of the Curie temperature distribution $\Delta T_{C}{ }^{0}=6.91 \pm 0.16 \mathrm{~K}$ from the data. We see that the experimental data follow Eq. (3) over nearly two orders of magnitude and the power-law exponent is determined to $\beta \cdot \delta=1.75 \pm 0.10$. In addition, we extract $\beta(\mathrm{H})$ from the individual data sets $\mathrm{M}\left(\mathrm{T}, \mathrm{H}=\mathrm{H}_{\mathrm{f}}\right)$ for the various magnetic fields. These data, displayed in Fig. 3b, allow an extrapolation of $\beta=0.3682 \pm 0.0033$ for the field free case. From this knowledge of $\beta$ and $\beta \cdot \delta$, we can then determine the critical exponent $\delta$, despite the fact that the phase transition is smeared out due to the $T_{C}$ distribution width. This demonstrates that the method of $\mathrm{M}(\mathrm{T}, \mathrm{H})$ measurements in conjecture with the $\Delta T_{C}(\mathrm{H})$ analysis allows an accurate determination of critical exponents even in inhomogeneous materials. The method does not require complex data analysis or specialized software, because it is based on two sequential data analysis steps, i.e. the $\mathrm{M}(\mathrm{T}, \mathrm{H})$ fits for a number of field values $\mathrm{H}$ and the $\Delta T_{C}(\mathrm{H})$ data analysis, which each contain a simple onedimensional fitting problem that can be solved in standard fashion.

Table 2 compares our values for the critical exponents $\beta, \delta$ and $\beta \cdot \delta$ with literature values for bulk materials as well as the 3D Heisenberg and Ising models [6]. We find agreement between our data and the Heisenberg model, as is appropriate for cubic samples. The comparison also shows that our method can compete with more sophisticated analysis methods based on scaling analysis, even though the precision for $\delta$ reported here is somewhat inferior [9]. The results demonstrate the feasibility of our 
method and illustrate its robustness towards intrinsic phase transition broadening, because the field induced phase transition broadening in our experiments is actually smaller than the intrinsic one, i.e. $\Delta T_{C}^{0}>\frac{\mu_{B} \cdot H}{k_{B}}$. This robustness is significant, because one has to keep in mind, that a scaling method would not allow a reliable data analysis for any of our samples, because such methods are based upon a single $\mathrm{T}_{\mathrm{C}}$ value. In particular, they cannot handle samples for which the zero field $\mathrm{T}_{\mathrm{C}}$ distributions play a significant role in the phase transition broadening, whereas our method works in the case of inhomogeneous samples exhibiting a substantial width of the $T_{C}$ distribution.

\section{ACKNOWLEDGEMENTS}

Work at Argonne is supported by the U. S. Department of Energy, Basic Energy Sciences - Materials Sciences under Contract W-31-109-ENG-38. Work at the Universidad del Valle is supported by COLCIENCIAS under contract No. 361-97. 


\section{Figure captions}

Fig. 1 Experimental M(T) data of an LCMO film for different values of the applied field $\mathrm{H}: 1 \mathrm{kOe}$ (circles) and $4 \mathrm{kOe}$ (squares) in comparison to the least-squares fitting results (lines) according to Eq. (1) (for clarity only a fraction of the actual data set is displayed). Inset: Magnetic field dependence of $\Delta \mathrm{T}_{\mathrm{C}}$; the individual $\mathrm{M}(\mathrm{T})$ fit results are shown as squares and the solid line is a least-squares fit of Eq. (3) to the $\Delta \mathrm{T}_{\mathrm{C}}(\mathrm{H})$ data set.

Fig. $2 \Delta \mathrm{T}_{\mathrm{C}}$ vs. field determined for three models: the mean-field approximation (closed squares), the 3D Ising model (open squares), and the 2D Ising model (closed circles).

Fig. 3 Plot of $\Delta T_{C}-\Delta T_{C}{ }^{0}$ and $\beta$ as a function of the applied field, derived from the individual $\mathrm{M}(\mathrm{T})$ data fits. The complete data sets are utilized to determine the critical exponents $\beta, \delta$ and $\beta \cdot \delta$, listed in Table 2 . 


\section{Tables}

Table 1: Comparison of the critical exponents for several theoretical models

\begin{tabular}{|l|l|l|l|c|}
\hline \multicolumn{1}{|c|}{ Model } & \multicolumn{1}{c|}{$\beta$} & \multicolumn{1}{c|}{$\delta$} & \multicolumn{1}{c|}{$\beta \cdot \delta$} & $\eta$ \\
\hline MFA & 0.5 & 3 & 1.5 & $1.50 \pm 0.02$ \\
\hline 2D Ising & 0.125 & 15 & 1.875 & $1.89 \pm 0.02$ \\
\hline 3D Ising [7] & $0.325 \pm 0.0015$ & $4.82 \pm 0.02$ & $1.567 \pm 0.013$ & $1.58 \pm 0.02$ \\
\hline
\end{tabular}

Table 2: Comparison of experimentally and theoretically determined values for critical exponents $\beta, \delta$, and $\beta \cdot \delta$.

\begin{tabular}{|l|l|l|l|}
\hline \multicolumn{1}{|c|}{ System } & \multicolumn{1}{c|}{$\boldsymbol{1}$} & \multicolumn{1}{c|}{$\boldsymbol{c}$} & \multicolumn{1}{c|}{$\beta \cdot \delta$} \\
\hline 3D Ising [7] & $0.325 \pm 0.0015$ & $4.82 \pm 0.02$ & $1.567 \pm 0.013$ \\
\hline 3D Heisenberg [8] & $0.3645 \pm 0.0025$ & $4.802 \pm 0.037$ & $1.750 \pm 0.026$ \\
\hline This work & $0.3682 \pm 0.0033$ & $4.76 \pm 0.32$ & $1.75 \pm 0.10$ \\
\hline LSMO-bulk [9] & $0.37 \pm 0.04$ & $4.25 \pm 0.2$ & $1.57 \pm 0.09$ \\
\hline Ni [3] & $0.395 \pm 0.010$ & $4.35 \pm 0.06$ & $1.718 \pm 0.068$ \\
\hline
\end{tabular}


${ }^{\dagger}$ permanent address: Departamento de Física, Universidad del Valle, Cali, A. A. 25360, Colombia

\section{References}

[1] See, for instance V. L. Pokrovskii, J. Magn. Magn. Mater. 200, 515 (1999); M. E. Fisher, Rev. Mod. Phys. 70, 653 (1998), A. Z. Patashinskii, and V. L. Pokrovskii, "Fluctuation Theory of Phase Transitions" (Pergamon, Oxford, 1979)

[2] N. Goldenfeld "Lectures on Phase Transitions and the Renormalization Group" (Addison Wesley, Reading, 1992)

[3] M. Seeger, S. N. Kaul, H. Kronmüller, and R. Reisser, Phys. Rev. B 51, 12585 (1995)

[4] G. Campillo, A. Berger, J. Osorio, J. E. Pearson, S. D. Bader, E. Baca and P. Prieto, J. Magn. Magn. Mater. 237, 61 (2001)

[5] C. Domb, Chapter 6 (p. 446-449) in "Phase Transitions and Critical Phenomena", Vol. 3, eds. C. Domb and M. S. Green (Academic Press, London, 1974)

[6] We have limited our numerical analysis to mean-field and Ising models only, because an accurate equation of state for the 3D Heisenberg model is not known, which is related to the existence of massless Goldstone modes for such isotropic spin systems; see, for instance: J. Berges, N. Tetradis and C. Wetterich, Phys. Rev. Lett. 77, 873 (1996)

[7] J. C. Le Guillou and J. Zinn-Justin, Phys. Rev. B 21, 3976 (1980)

[8] K. Chen, A. M. Ferrenberg, and D. P. Landau, J. Appl. Phys. 73, 5488 (1993)

[9] K. Ghosh, C. J. Lobb, R. L. Greene, S. G. Karabashev, D. A. Shulyatev, A. A. Arsenov, and Y. Mukovskii, Phys. Rev. Lett. 81, 4740 (1998) 


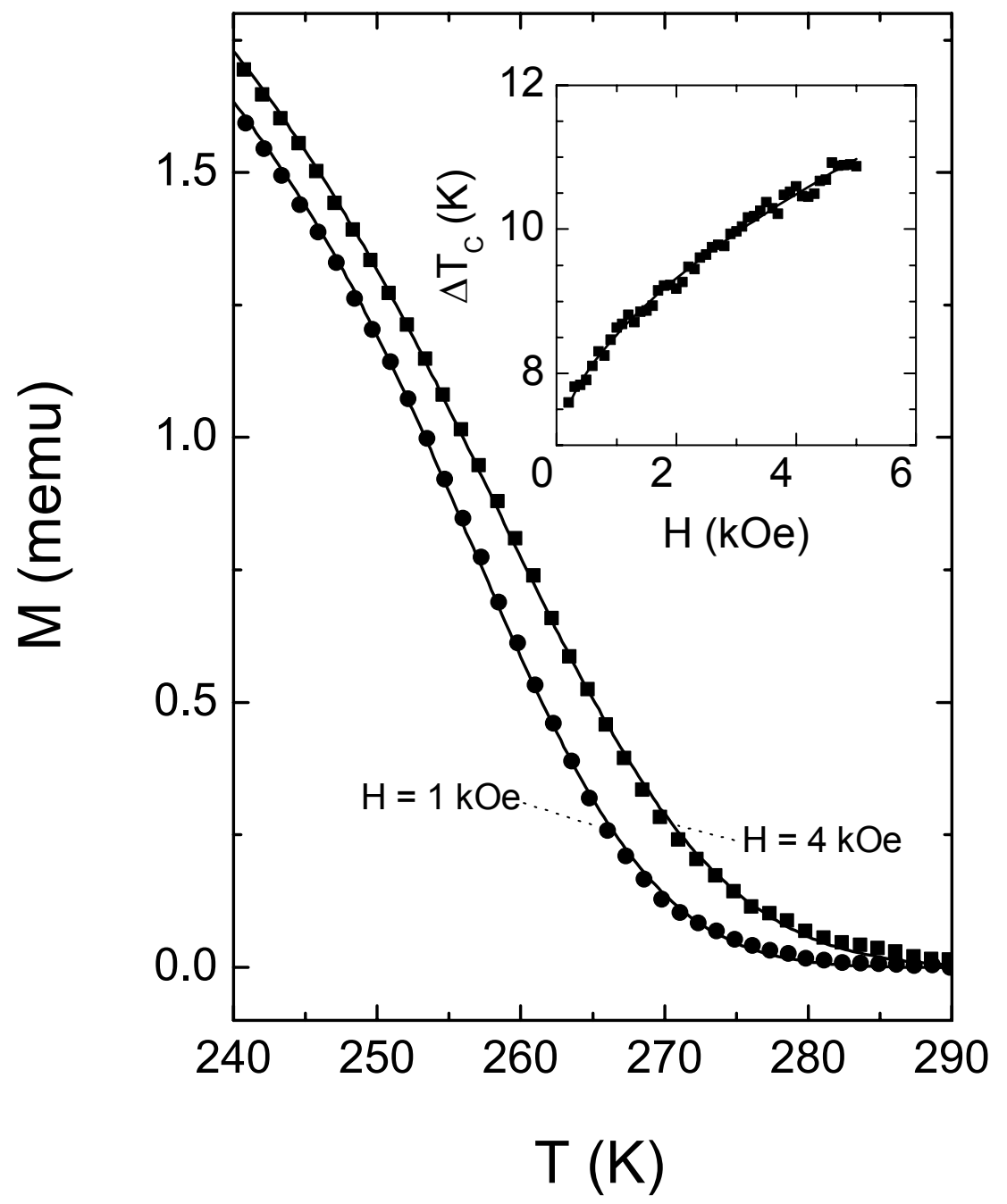

Figure 1 


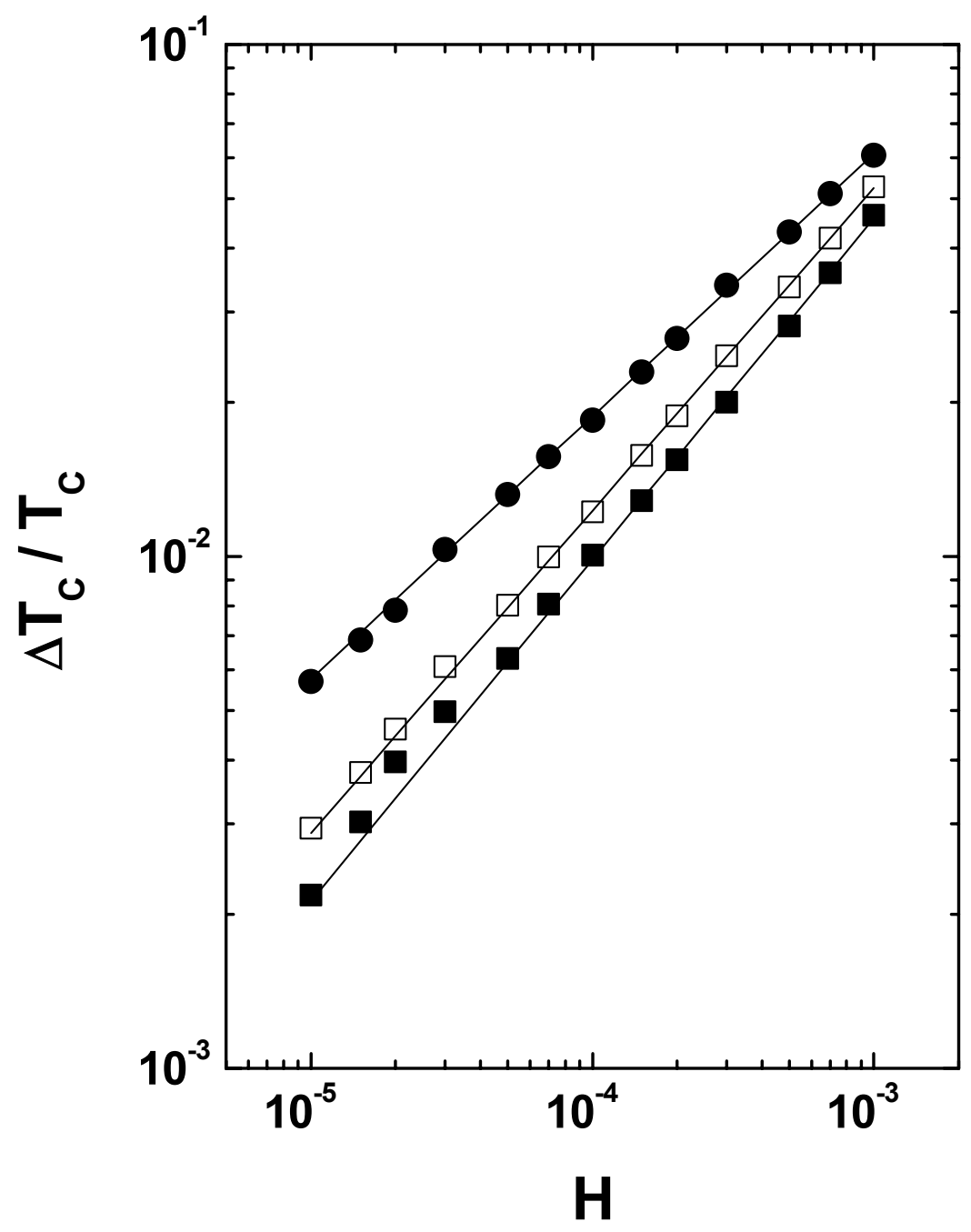

Figure 2 

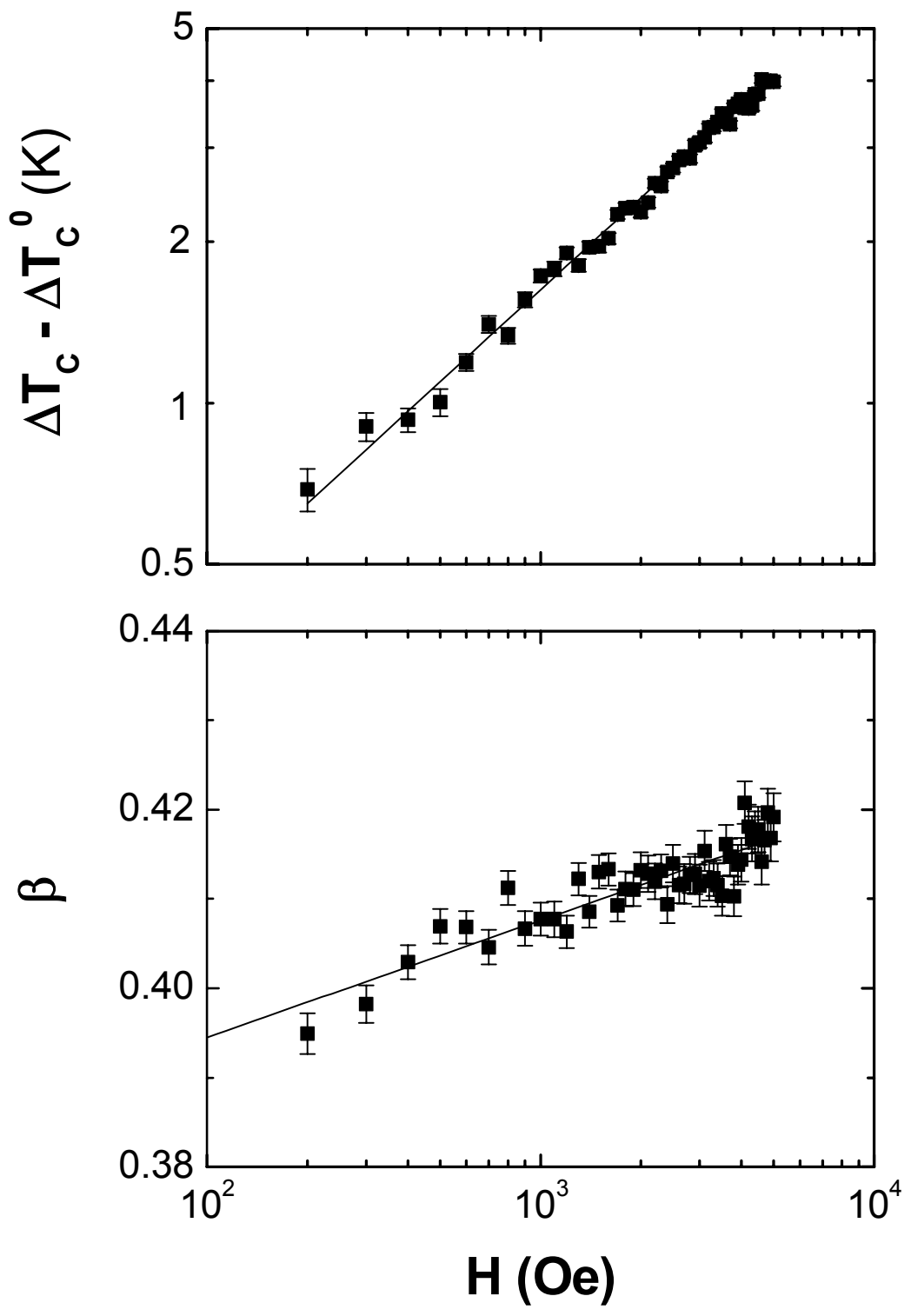

Figure 3 